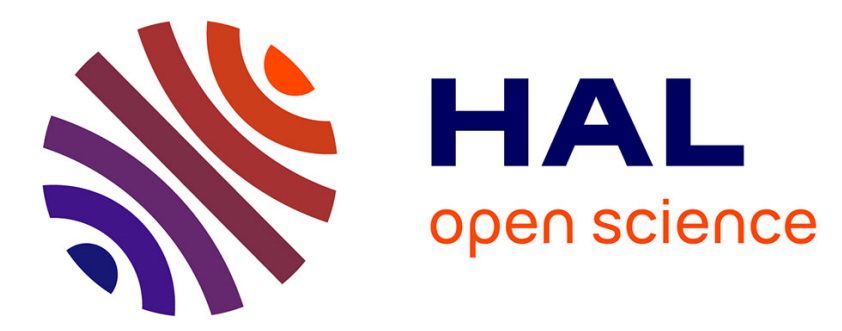

\title{
Robust Microscale Grasping Using a Self Scheduled Dynamic Controller.
}

Mokrane Boudaoud, Marcelo Gaudenzi de Faria, Yassine Haddab, Sinan

Haliyo, Yann Le Gorrec, Philippe Lutz, Stéphane Régnier

\section{To cite this version:}

Mokrane Boudaoud, Marcelo Gaudenzi de Faria, Yassine Haddab, Sinan Haliyo, Yann Le Gorrec, et al.. Robust Microscale Grasping Using a Self Scheduled Dynamic Controller.. 19th IFAC World Congress, Aug 2014, Cape Town, South Africa. 10.3182/20140824-6-ZA-1003.00413 . hal-03221855

\section{HAL Id: hal-03221855 \\ https://hal.science/hal-03221855}

Submitted on 10 May 2021

HAL is a multi-disciplinary open access archive for the deposit and dissemination of scientific research documents, whether they are published or not. The documents may come from teaching and research institutions in France or abroad, or from public or private research centers.
L'archive ouverte pluridisciplinaire HAL, est destinée au dépôt et à la diffusion de documents scientifiques de niveau recherche, publiés ou non, émanant des établissements d'enseignement et de recherche français ou étrangers, des laboratoires publics ou privés. 


\title{
Robust Microscale Grasping Using a Self Scheduled Dynamic Controller
}

\author{
Mokrane Boudaoud* Marcelo Gaudenzi De Faria** \\ Yassine Haddab ${ }^{* *}$ Sinan Haliyo* Yann Le Gorrec** \\ Philippe Lutz** Stéphane Régnier* \\ * Institut des Systèmes Intelligents et de Robotique, Université Pierre et Marie \\ Curie, CNRS UMR 7222, 4 Place Jussieu, F-75252 Paris Cedex, France \\ ** FEMTO-ST Institute, UMR CNRS 6174 - UFC-ENSMM-UTBM; Automatic \\ Control and Micro-Mechatronic Systems (AS2M) Department; 24, rue Alain \\ Savary, 25000 Besançon - France
}

\begin{abstract}
:
This paper deals with robust gripping force control at the microscale for a safe manipulation of deformable soft materials. Since mechanical properties of micrometer sized objects are uncertain, instability often occurs during a gripping task. In this article, the design of an output feedback self-scheduled dynamic controller is proposed considering parametric uncertainties of a set of 65 soft and resilient microspheres. The degrees of freedom of the controller are managed by the design of a set of elementary observers. Robustness with respect to parametric uncertainties is satisfied thanks to an iterative procedure based on an eigenstructure assignment methodology and a worst case analysis. The developed controller is of low order and can be implemented in real time. Simulations demonstrate the validity of the proposed control approach.
\end{abstract}

Keywords: Micro and Nano Mechatronic Systems, Force feedback, robust control, grasping

\section{INTRODUCTION}

Microrobotics hold promises for efficient and safe manipulation of biologic samples and living cells (Liu et al. [2009]). In precursor works, Atomic Force Microscopy inspired techniques has proven themselves a reliable tool for characterization of biomaterials. Muller et al. [2009] used an AFM probe to obtain high resolution force images of living cells. Boukallel et al. [2009] proposed an improved probe design to measure mechanical characteristics of cells in long traction/compression cycles. Desmaele et al. [2012] introduced a dynamic measurement method improving the reliability. However, the single cantilever or similar design of such sensors are ill adapted for manipulation. Recently, the development of microgrippers that include both actuation and force sensing in dimensions adapted to biologic samples opens the way to novel and cost-effective applications ranging from in-vitro fertilization to genetics (Carrozza et al. [2000], Beyeler et al. [2007]).

Biologic cells are highly deformable soft materials. They are very sensitive on applied force and on how they are handled. The use of grippers for their manipulation calls for a precise force control of grasping. This particular issue is evidently not limited to biologic samples and is a general concern for micro and nanoscale manipulation. To apply safety gripping forces required for the manipulation of soft objects, several solutions have been reported. Bolopion et al. [2012] uses haptic feedback allowing the user to interact with microscale objects and leaves the force control to the operator. In a more traditional approach, feedback control (Liu et al. [2009], Carrozza et al. [2000], Park et al. [2005]) allow for an automated approach to gripping.

At the microscale, soft materials have mechanical properties, namely stiffness and damping, close to that of actuation and sensing systems of microgrippers. Therefore, during gripping tasks, samples have enough variation to induce instabilities to damage the gripper or the sample. In the literature, microscale force feedback control design are most of time based on PI, PID or LQG schemes (Liu et al. [2009], Carrozza et al. [2000], Park et al. [2005], Boudaoud et al. [2013]). Controller synthesis is often achieved considering the mechanical properties of a single sample and closed loop performance are validated experimentally when gripping the sample used for the synthesis (Liu et al. [2009], Boudaoud et al. [2013]). These approaches lack the robustness required for micromanipulation. To overcome this problem, $\mathrm{H}_{\infty}$ controllers such as proposed in Rakotondrabe and Le Gorrec [2010], Rakotondrabe et al. [2007] are often used. Resulting schemes allow for a robust force control, but such controllers are often of high order and difficult to implement in real time (Boudaoud et al. [2012], Poussot-Vassal et al. [2008]).

In the aim to provide robustness in a low-order controller an approach is proposed here. Its synthesis is based on an eigenstructure assignment methodology as previously mentioned in Boudaoud et al. [2012]. The shortcoming of this approach is in the presence of unknown variable parameters. Indeed, in a gripping case, it is assumed that size, stiffness and the damping of the samples are part of those unknowns. To overcome this problem, based on 


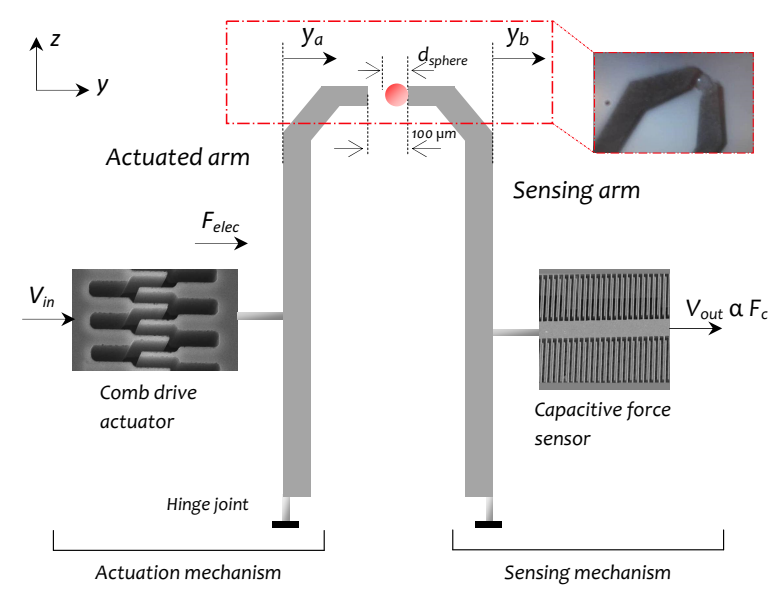

Fig. 1. Simplified scheme of the FT-G100 microgripper.

a set of elementary observers, multi-model assignment constraints are defined here using an iterative procedure (Magni et al. [1998]). The most relevant multimodel constraints are defined considering parametric uncertainties in a set of 65 soft and resilient microspheres with diameters ranging from $40 \mu m$ to $80 \mu m$ and stiffness from $2,8 \mathrm{~N} / \mathrm{m}$ to $15,7 \mathrm{~N} / \mathrm{m}$. The order of the controller is equal to the number of observers, and is potentially low.

This proposed approach is implemented here using an electrostatic microgripper with an integrated force sensor. A nonlinear coupled model of a microgripper grasping an object is established. A set of 65 microspheres with varying size, stiffness and damping are mechanically characterized. Using the nonlinear model and the sample dataset, gripping force control is then achieved through the above mentioned methodology.

\section{SPECIFICATIONS OF THE MICROGRIPPER AND SAMPLE MICROSPHERES}

The microgripper used in this study is the FT-G100 from Femto Tools GmbH (Beyeler et al. [2007]). A detailed description of its architecture and working principle are described in Boudaoud et al. [2013]. This two finger microgripper depicted in fig. 1 includes comb-drive actuation on one digit and capacitive force sensing on the other. The gap of the gripper is $100 \mu \mathrm{m}$.

Manipulated samples are thermoplastic particles, called expancel microspheres, provided by AkzoNobel ${ }^{1}$. They are composed of a polymer shell encapsulating a gas. They are deformable, soft and resilient, with properties in the range of biological samples which makes them very attractive for force control experiments at the microscale. Three models of microspheres have been used: 461 WE 20 d36, 461 DE 20 d70 and 461 DET 40 d25. For each model, the manufacturer provides the size, with around $10 \%$ certitude as shown in Table 1, but the stiffness and the damping are not known. Hence, the size and both the stiffness and the damping of a set of 65 microspheres are experimentally identified in section 3.2 using the FT-G100 microgripper .

1 http://www.akzonobel.com
Table 1. Reference, diameter and number of characterized Expancel microspheres.

\begin{tabular}{lll}
\hline Reference & Diameter & Number of samples \\
\hline 461 WE $20 \mathrm{~d} 36$ & $57 \mu \mathrm{m} \pm 14 \mu \mathrm{m}$ & 25 \\
\hline $461 \mathrm{DE} 20 \mathrm{~d} 70$ & $55 \mu \mathrm{m} \pm 5 \mu \mathrm{m}$ & 17 \\
\hline $461 \mathrm{DET} 40 \mathrm{~d} 25$ & $68 \mu \mathrm{m} \pm 10 \mu \mathrm{m}$ & 23 \\
\hline
\end{tabular}

\section{NONLINEAR MODELING OF THE GRASPING}

\subsection{Modeling of the gripper}

In Boudaoud et al. [2012], a nonlinear model of the FTG100 actuation mechanism (fig. 1) is described along with an experimental validation. It's a mass-spring-damper model including the stiffness $k_{a}$ and the damping $d_{a}$ that are nonlinear polynomial functions of the position of the actuated $\operatorname{arm} y_{a}$ :

$$
k_{a}\left(y_{a}\right)=\sum_{i=1}^{6} k_{i a} y_{a}^{i-1} \quad \text { and } \quad d_{a}\left(y_{a}\right)=\sum_{i=1}^{4} d_{i a} y_{a}^{i}
$$

This mass-spring-damper model is extended to the case where a microsphere is gripped between the actuated and sensing arms. This model is obtained by coupling both the nonlinear model of the actuation mechanism with the linear mass-spring-damper model of the sensing mechanism and considering the gripped object as a springdamper with a particular stiffness $k_{o}$ and damping $d_{o}$.

$$
\begin{aligned}
{\left[\begin{array}{l}
\dot{y}_{a} \\
\ddot{y}_{a} \\
\dot{y}_{b} \\
\ddot{y}_{b}
\end{array}\right]=} & {\left[\begin{array}{cccc}
0 & 1 & 0 & 0 \\
-\frac{k_{o}+k_{a}\left(y_{a}\right)}{m_{a}} & -\frac{d_{o}+d_{a}\left(y_{a}\right)}{m_{a}} & \frac{k_{o}}{m_{a}} & \frac{d_{o}}{m_{a}} \\
0 & 0 & 0 & 1 \\
\frac{k_{o}}{m_{b}} & \frac{d_{o}}{m_{b}} & -\frac{k_{o}+k_{b}}{m_{b}} & -\frac{d_{o}+d_{b}}{m_{b}}
\end{array}\right]\left[\begin{array}{c}
y_{a} \\
\dot{y}_{a} \\
y_{b} \\
\dot{y}_{b}
\end{array}\right] } \\
+ & {\left[\begin{array}{l}
0 \\
\frac{N_{a} \varepsilon h_{z}}{2 m_{a} g D_{a}} \\
0 \\
0
\end{array}\right] V_{i n}^{2} } \\
F_{c}= & {\left[\begin{array}{lll}
k_{o} & d_{o}-k_{o}-d_{o}
\end{array}\right]\left[\begin{array}{c}
y_{a} \\
\dot{y}_{a} \\
y_{b} \\
\dot{y}_{b}
\end{array}\right] }
\end{aligned}
$$

where $y_{i}, k_{i}, d_{i}$ and $m_{i}$ are respectively the position, stiffness, damping and mass of the actuated arm $(i \Leftrightarrow$ $a)$ and the sensing arm $(i \Leftrightarrow b)$ as depicted in fig. 1 . $N_{a}, \varepsilon, h_{z}$, and $g$ are intrinsic parameters of the comb drive, providing the electrostatic force $F_{\text {elec }}\left(V_{i n}\right) . F_{c}$ is the gripping force, as measured through the capacitive sensor. $D_{a}$ is an amplification parameter. Numerical values of the model parameters are given in Boudaoud et al. [2012] and Boudaoud et al. [2013].

\subsection{Experimental characterization of the microspheres}

In a random gripping case, the stiffness $k_{o}$, the damping $d_{o}$ and the diameter $d_{\text {sphere }}$ (fig. 1) of the gripped object are unknown. A robust control strategy would ideally be able to deal with these uncertainties. For the synthesis of such a controller, a set of 65 expancel microspheres with varying $k_{o}, d_{o}$ and $d_{\text {sphere }}$ are experimentally identified. 

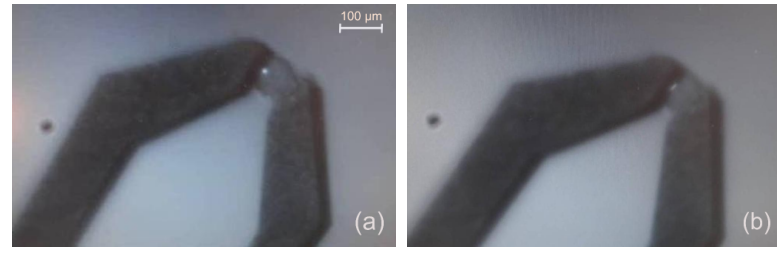

Fig. 2. The microgripper handling an expancel microsphere: uncompressed (a) and compressed (b).

This dataset is then used in the study, first for synthesis and then for validation.

The set of microspheres are characterized using solely the microgripper, which was mounted on a 3 DoF stage for this purpose. The user positions the gripper on the sphere manually until it contacts the sensing arm as depicted in fig. 1. In order to contact both fingers on the object, the actuated arm of the gripper is then fed with an input voltage $V_{i n 0}$ until the sensing arm registers a signal. At this stage, the gripping force is lower than $0.1 \mu N$. The final position $y_{a}$ of the actuated arm is measured with an external laser interferometer (SP-120 SIOS Meßtechnik $\mathrm{GmbH})$. The $d_{\text {sphere }}$ is deduced from this measurement (i.e. $\left.d_{\text {sphere }}=100 \mu m-y_{a}\right)$.

A $10 V$ step signal is then applied to the actuator, which compresses the grasped sample (Fig.2). The step response provided by the force sensor is hence used for the identification of the stiffness $k_{0}$ and the damping $d_{0}$ using the nonlinear coupled model as in eq. (1).

Size and stiffness identification results are shown in Fig.3. $d_{\text {sphere }}$ ranges from $43.66 \mu \mathrm{m}$ to $78.11 \mu \mathrm{m}$ and $k_{o}$ varies between $2.8 \mathrm{~N} / \mathrm{m}$ and $15.7 \mathrm{~N} / \mathrm{m}$. The damping $d_{o}$ ranges from $1.5 \times 10^{-6}$ to $1.8 \times 10^{-4}$. The first eigenmode of the coupled system (grasping model) is between $1.6 \mathrm{kHz}$ and $2 \mathrm{kHz}$. The frequency response of the coupled system to a $10 \mathrm{~V}$ step voltage is shown in Fig.4 for three different microspheres from Table 1.

\subsection{Linearization of the grasping model}

For each object in the dataset, the nonlinear grasping model is linearized in the neighborhood around an operating point $\delta_{a}$. For the comb drive actuator, the nonlinear parameters $k_{a}\left(\delta_{a}\right)$ and $d_{a}\left(\delta_{a}\right)$ are polynomial. The governing parameter $\delta_{a}$ is the position of the actuated arm. Using a Jacobian linearization, the nonlinear plant of eq. (1) is formulated into a linear parameter varying model:

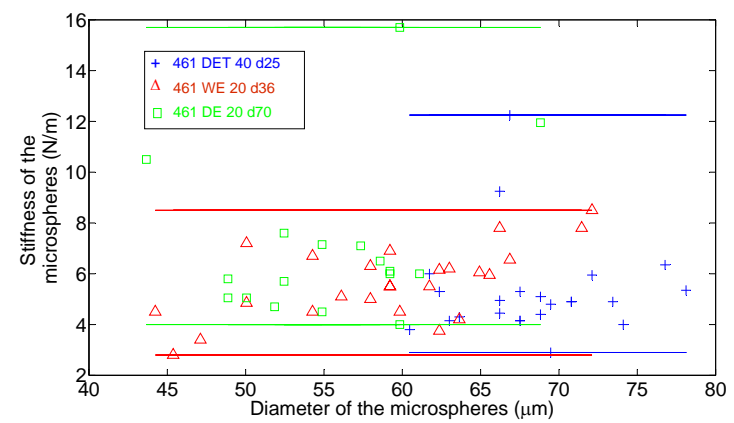

Fig. 3. Disparity map of diameter and stiffness of characterized Expancel microspheres.
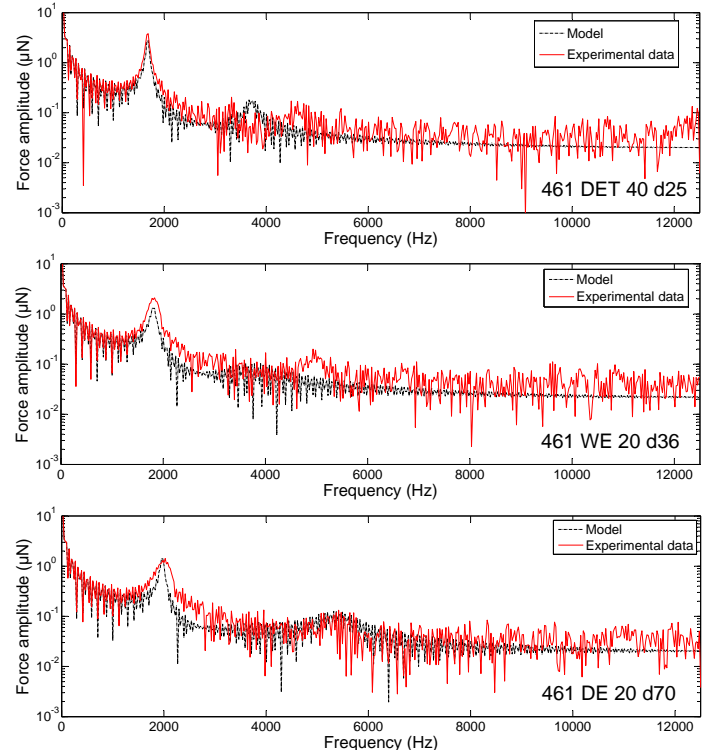

Fig. 4. Frequency responses of the microgripper handling three different microspheres for a $10 \mathrm{~V}$ step voltage - simulation and experimental data.

$$
\begin{gathered}
G_{c}\left(\Delta_{c}\right)\left\{\begin{array}{l}
\dot{X}_{c}=A_{c}\left(\Delta_{c}\right) X_{c}+B_{c} U \\
F_{c}=C_{c}\left(\Delta_{c}^{\prime}\right) X_{c}
\end{array}\right. \\
A_{c}=\left[\begin{array}{cccc}
0 \\
-\frac{k_{o}+\sum_{i=1}^{6} i k_{i a} \delta_{a}^{i-1}}{m_{a}}-\frac{d_{o}+\sum_{i=0}^{4} d_{i a} \delta_{a}^{i}}{m_{a}} & \frac{k_{o}}{m_{a}} & \frac{d_{o}}{m_{a}} \\
0 & 0 & 0 & 1 \\
\frac{k_{o}}{m_{b}} & \frac{d_{o}}{m_{b}} & -\frac{k_{o}+k_{b}}{m_{b}} & -\frac{d_{o}+d_{b}}{m_{b}}
\end{array}\right] \\
B_{c}=\left[\begin{array}{cccc}
0 & \frac{N_{a} h_{z}}{2 m_{a} g D_{a}} & 0 & 0
\end{array}\right]^{T} C_{c}=\left[\begin{array}{ccc}
k_{o} & d_{o} & -k_{o}-d_{o}
\end{array}\right] \\
X_{c}=\left[\begin{array}{lll}
\tilde{y}_{a} \dot{\tilde{y}}_{a} & \tilde{y}_{b} & \dot{\tilde{y}}_{b}
\end{array}\right]^{T}
\end{gathered}
$$

where $A_{c} \in \mathbb{R}^{n \times n}, B_{c} \in \mathbb{R}^{n \times m}$ and $C_{c} \in \mathbb{R}^{p \times n}$, with $n=4, m=1$ and $p=1 . \Delta_{c}=\left[\begin{array}{ll}\Delta_{c}^{\prime} & d_{\text {sphere }}\end{array}\right]$ and $\Delta_{c}^{\prime}=\left[\begin{array}{ll}k_{o} & d_{o}\end{array}\right] . \quad \tilde{y}_{a}$ is the variation of $y_{a}$ around the operating point $\delta_{a}$. The nonlinearity arising from the square voltage is overcome by considering $U=V_{i n}^{2}$ as the input of the model for the controller design. This consideration is valid since the input voltage applied to the actuator is always positive.

A set of 65 Linear Time Invariant (LTI) models are derived from eq. (2). Stiffness $k_{o}$, damping $d_{o}$ and the diameter $d_{\text {sphere }}$ (expressed as $100 \mu m-\delta_{a}$ ) are matched for each object in the dataset. Bode diagram of the elementary LTI models obtained from eq. (2) are shown in Fig.5.

The capacitive force sensor provides its output with a delay $T_{r}=0.3 \mathrm{~ms}$. This delay has to be taken into account

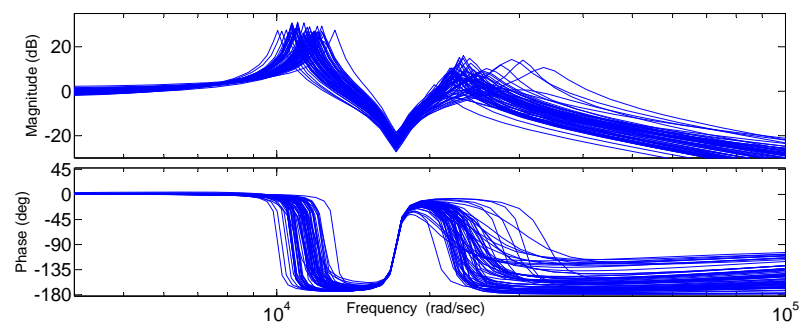

Fig. 5. Bode diagrams of the set of LTIs models. 
for the controller synthesis. As such, let us consider $F_{c d}$ the delayed gripping force signal. The delay is added to the model of eq. 2 by means of a first order Padé approximation as follows:

$$
G_{\text {delay }}=\frac{F_{c d}}{F_{c}}=e^{-T r s} \simeq \frac{1-\frac{T_{r}}{2} s}{1+\frac{T_{r}}{2} s}
$$

The order of the state space model is therefore increased by 1 . Considering $A_{\text {delay }}, B_{\text {delay }}, C_{\text {delay }}$ and $D_{\text {delay }}$ the state space matrices of $G_{\text {delay }}$ under the state space representation, the LPV model becomes:

$$
\begin{gathered}
G_{c d}\left(\Delta_{c}\right)\left\{\begin{array}{l}
\dot{X}_{c d}=A_{c d}\left(\Delta_{c}\right) X_{c d}+B_{c d} U \\
F_{c d}=C_{c d}\left(\Delta_{c}^{\prime}\right) X_{c d}
\end{array}\right. \\
A_{c d}\left(\Delta_{c}\right)=\left[\begin{array}{cc}
A_{c}\left(\Delta_{c}\right) & 0_{n \times 1} \\
B_{\text {delay }} C_{c}\left(\Delta_{c}^{\prime}\right) & A_{\text {delay }}
\end{array}\right], B_{c d}=\left[\begin{array}{l}
B_{c} \\
0_{1 \times m}
\end{array}\right] \\
C_{c d}\left(\Delta_{c}^{\prime}\right)=\left[\begin{array}{ll}
D_{\text {delay }} C_{c}\left(\Delta_{c}^{\prime}\right) & C_{\text {delay }}
\end{array}\right] \\
X_{c d}=\left[\begin{array}{ll}
X_{c}^{T} & X_{d}^{T}
\end{array}\right]^{T}
\end{gathered}
$$

where $X_{d} \in \mathbb{R}^{1 \times 1}$ is the state of the first order Padé approximation $G_{\text {delay }}$.

\section{SELF SCHEDULED CONTROLLER}

Control specifications are defined in terms of precision, closed loop bandwidth and damping as required for a micromanipulation task. For nominal performance, control specifications are given as: (i) The closed loop response time of the system must be lower than $15 \mathrm{~ms}$. (ii) No overshoot is admitted. An overshoot of a few $\mu N$ can destroy the manipulated object. (iii) The maximum static error must be lower than $0.1 \%$.

The proposed control strategy is based on an output feedback eigenstructure assignment $(E S A)$. The control strategy is first build considering a nominal model, a configuration for a given fixed value of $\Delta_{c}$. In a second step to ensure the robustness, $\Delta_{c}$ is considered variable. An iterative strategy and a worst-case analysis is used to tune the parameters of the multimodel eigenstructure assignment.

\subsection{ESA with fixed $\Delta_{c}=\Delta_{c 1}$}

The classical ESA methodology is first proposed in Moore and Klein [1976]. Let us consider the coupled model (grasping model) $\left[A_{c d}\left(\Delta_{c 1}\right), B_{c d}, C_{c d}\left(\Delta_{c 1}^{\prime}\right)\right]$ with a fixed parameter $\Delta_{c}=\Delta_{c 1}$.

Lemma 1. Given an eigenvalue $\lambda_{i}\left(\Delta_{c 1}\right)$, the triple $\Gamma=$ $\left(\lambda_{i}\left(\Delta_{c 1}\right), \nu_{i}\left(\Delta_{c 1}\right), \omega_{i}\left(\Delta_{c 1}\right)\right)$ satisfying

$$
\left[A_{c d}\left(\Delta_{c 1}\right)-\lambda_{i}\left(\Delta_{c 1}\right) I_{n+1}-B_{c d}\right]\left[\begin{array}{l}
\nu_{i}\left(\Delta_{c 1}\right) \\
\omega_{i}\left(\Delta_{c 1}\right)
\end{array}\right]=0
$$

is assigned by the static gain $K_{c}$ if and only if

$$
K_{c} C_{c d}\left(\Delta_{c 1}^{\prime}\right) \nu_{i}\left(\Delta_{c 1}\right)=\omega_{i}\left(\Delta_{c 1}\right)
$$

where $\nu_{i}\left(\Delta_{c 1}\right) \in \mathbb{C}^{n+1}$ and $\omega_{i}\left(\Delta_{c 1}\right) \in \mathbb{C}^{m}$ are respectively the eigenvector and the input direction of the closed loop system.

This control strategy has two main limitations: (i) the degrees of freedom of the controller (i.e. number of eigenstructures that can be assigned in closed loop) is limited by the number of outputs $p$ of the system, (ii) the controller is generally not robust against the variation of uncertain and nonlinear parameters.

The later issue is addressed in the next section. For the former, considering the LPV model of eq. 4, the number of eigenvalues that can be assigned is $n+1=5$ while the number of output of the system is one $(p=1)$. Therefore, to offer additional degrees of freedom required for the simultaneous resolution of linear constraints (6) where eigenvectors $\nu_{i}\left(\Delta_{c 1}\right)\left(1<i<r_{1}\right.$ with $r_{1}=$ the number of multimodel constraints) are distinct, it is necessary to increase the number of outputs.

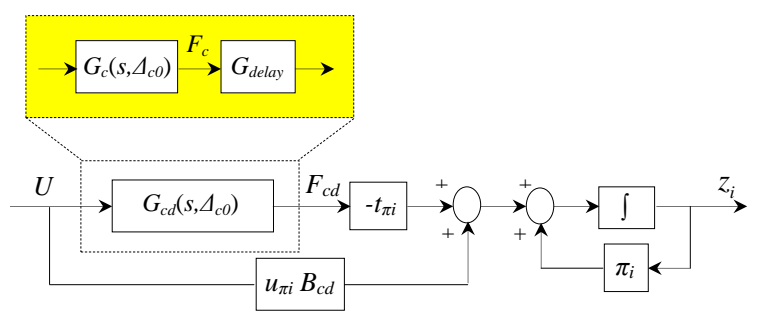

Fig. 6. Elementary observer of $z_{i}=u_{\pi_{i}} X_{c d}$.

As such, to assign $r_{1}$ eigenvalues to the fixed parameter system of eq. 4 , a set of $n_{c}\left(n_{c}=r_{1}-p\right)$ observers are added:

Lemma 2. The system defined by (see Fig.6)

$$
\begin{aligned}
& \frac{d z_{i}}{d t}=\pi_{i} z_{i}-t_{\pi_{i}} F_{c d}+u_{\pi_{i}} B_{c d} U \\
& u_{\pi_{i}} A_{c d}\left(\Delta_{c 1}\right)+t_{\pi_{i}} C_{c d}\left(\Delta_{c 1}^{\prime}\right)=\pi_{i} u_{\pi_{i}}
\end{aligned}
$$

where, $u_{\pi_{i}} \in \mathbb{C}^{1 \times(n+1)}, t_{\pi_{i}} \in \mathbb{C}^{1 \times p}$ and $\pi_{i} \in \mathbb{C}^{1 \times 1}$

is an observer of the variable $z_{i}=u_{\pi_{i}} X_{c d}$ and the observation error $\varepsilon_{i}=z_{i}-u_{\pi_{i}} X_{c d}$ satisfies $\frac{\partial \varepsilon_{i}}{\partial t}=\pi_{i} \varepsilon_{i}$.

This Lemma highlights that a linear relation of the states $u_{\pi_{i}} X_{c d}$ can be estimated by an observer. This increases the number of outputs and offers additional degrees of freedom to the controller. It then allows assigning as many additional triples as the number of observations.

For $n_{c}$ observers, the following notations are considered:

$$
U_{o} A_{c d}\left(\Delta_{c 1}\right)+T_{o} C_{c d}\left(\Delta_{c 1}^{\prime}\right)=\Pi_{o} U_{o}
$$

with :

$$
\begin{gathered}
U_{o}=\left[\begin{array}{l}
u_{\pi 1} \\
\vdots \\
u_{\pi_{n c}}
\end{array}\right] T_{o}=\left[\begin{array}{l}
t_{\pi 1} \\
\vdots \\
t_{\pi_{n c}}
\end{array}\right] \prod_{o}=\left[\begin{array}{ccc}
\pi 1 & \cdots & 0 \\
\vdots & \ddots & \vdots \\
0 & \cdots & \pi_{n c}
\end{array}\right] \\
Z=\left[\begin{array}{lll}
z_{1} & \ldots & z_{n c}
\end{array}\right]^{T}
\end{gathered}
$$

where $U_{o} \in \mathbb{C}^{n_{c} \times(n+1)}, T_{o} \in \mathbb{C}^{n_{c} \times p}$ and $\Pi_{o} \in \mathbb{C}^{n_{c} \times n_{c}}$.

Therefore, the control problem consists now in finding a gain matrix $K_{c}=\left[\begin{array}{ll}K_{y} & K_{z}\end{array}\right]$ such that the system

$$
\left\{\begin{array}{l}
\dot{X}_{c d}=A_{c d}\left(\Delta_{c 1}\right) X_{c d}+B_{c d} U \\
\frac{d Z}{d t}=\Pi_{o} Z-T_{o} F_{c d}+U_{o} B_{c d} U \\
F_{c d}=C_{c d}\left(\Delta_{c 1}^{\prime}\right) X_{c d}
\end{array}\right.
$$

controlled by the input

$$
U=-K_{y} F_{c d}-K_{z} Z
$$




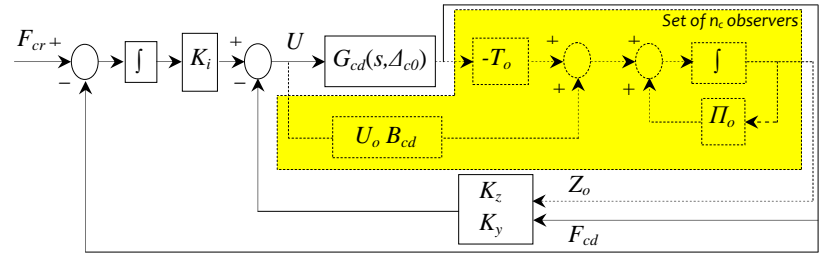

Fig. 7. Self-scheduled control scheme with the set of observers.

has the expected performance.

In the study, we consider the control problem by taking into account the separation principle. It is equivalent to assign (with a static gain $K_{c}=\left[K_{y} K_{z}\right]$ ) the eigenvalues of the system (10) and that of the system (12).

$$
\left\{\begin{array}{l}
\dot{X}_{c d}=A_{c d}\left(\Delta_{c 1}\right) X_{c d}+B_{c d} U \\
{\left[\begin{array}{l}
F_{c d} \\
Z
\end{array}\right]=\left[\begin{array}{l}
C_{c d}\left(\Delta_{c 1}^{\prime}\right) \\
U_{o}
\end{array}\right] X_{c d}}
\end{array}\right.
$$

The advantage of choosing system (12) comes from its structure, which allows defining explicitly the variables $F_{c d}$ and $Z$ as the outputs.

Moreover, in order to satisfy the closed loop precision specification, this model is augmented by an integrator. The control law as depicted by in Fig. 7 is now :

$$
U=K_{i} \int\left(F_{c r}-F_{c d}\right)-K_{y} F_{c d}-K_{z} Z
$$

where $K_{i}$ is the gain of the integrator. The controller's gain is expressed as $K_{c}=\left[\begin{array}{lll}K_{y} & K_{z} & K_{i}\end{array}\right]$

The order of the open loop system now equals to five and $p+1+n_{c}$ measurements are hence available: the output of the system $F_{c d}$, the signal $F_{c r}-F_{c d}$ and the outputs of the observer $Z$.

\subsection{ESA with variable $\Delta_{c}$}

The output feedback controller satisfies required closed loop performance only for $\Delta_{c}=\Delta_{c 1}$. Hence, robustness is no longer guaranteed for the entire set of LTI models. Let us now assume that in the set of $q$ LTI models, $r_{i}$ eigenstructures must be assigned for each model $G_{c d}\left(\Delta_{c i}\right)$. Therefore $\sum_{i=1}^{q} r_{i}$ linear constraints must be satisfied.

$$
\begin{gathered}
\left(A_{c d}\left(\Delta_{c 1}\right)-\lambda_{1}\left(\Delta_{c 1}\right) I_{n+1}\right) \nu_{1}\left(\Delta_{c 1}\right)-B_{c d} \omega_{1}\left(\Delta_{c 1}\right)=0 \\
\vdots \\
\left(A_{c d}\left(\Delta_{c q}\right)-\lambda_{r_{q}}\left(\Delta_{c q}\right) I_{n+1}\right) \nu_{r_{q}}\left(\Delta_{c q}\right)-B_{c d} \omega_{r_{q}}\left(\Delta_{c q}\right)=0
\end{gathered}
$$

Multimodel constraints are therefore defined by :

$$
\begin{gathered}
K_{c} C_{c d}\left(\Delta_{c 1}^{\prime}\right) \nu_{1}\left(\Delta_{c 1}\right)=\omega_{1}\left(\Delta_{c 1}\right) \\
\vdots \\
K_{c} C_{c d}\left(\Delta_{c q}^{\prime}\right) \nu_{r_{q}}\left(\Delta_{c q}\right)=\omega_{r_{q}}\left(\Delta_{c q}\right)
\end{gathered}
$$

The output feedback gain $K_{c}$ must be defined to satisfy this set of multimodel constrains. If the $q$ LTI models correspond to worst-case models, the multimodel synthesis leads to an output feedback controller that meets the robustness performance and the closed loop stability in this set. A solution to satisfy eq. (15) is to schedule the static gain $K_{c}$ by an interpolation formula and to use $\Delta_{c i}$ as the scheduling variable. However, here uncertain parameters, namely the stiffness, the damping and the diameter of the microspheres, are not directly accessible.
A self scheduled controller is hence proposed as follows: Proposition 1: Given $U_{o} \in \mathbb{C}^{n_{c} \times(n+1)}, T_{o} \in \mathbb{C}^{n_{c} \times p}$ and $\Pi_{o} \in \mathbb{C}^{n_{c} \times n_{c}}$ satisfying eq. (9) such that the interconnection between $\left\{\pi_{1}, \ldots, \pi_{n c}\right\}$ and $\left\{\lambda_{1}\left(\Delta_{c 1}\right) \ldots\right.$, $\left.\lambda_{r_{1}}\left(\Delta_{c 1}\right), \ldots, \lambda_{1}\left(\Delta_{c q}\right), \ldots, \lambda_{r_{q}}\left(\Delta_{c q}\right)\right\}$ is an empty set. For each assignment, a vector $\gamma_{j}\left(\Delta_{c i}\right) \quad(1<\mathrm{j}<$ number of assigned eigenstructures) related to a given LTI model $G_{c d}\left(\Delta_{c i}\right)$ is defined by eq. (16). The dynamic controller satisfies the set of equations (15) if and only if eq. (17) is satisfied where $K_{c}=\left[\begin{array}{lll}K_{y} & K_{z} & K_{i}\end{array}\right]$.

In order to define the multimodel constraints, an iterative procedure is performed as follows:

Step 1: Initialization Designing an output feedback controller (through Proposition 1) with a set of observers on a nominal model $G_{c d}\left(\Delta_{c 1}\right)$. At this step, any fixed value of $\Delta_{c}=\Delta_{c 1}$ can be used from the set of the 65 LTI models. The gain $K_{c}$ is designed considering the following multimodel constraints:

$$
\begin{gathered}
K_{c} C_{c d}\left(\Delta_{c 1}^{\prime}\right) \nu_{1}\left(\Delta_{c 1}\right)=\omega_{1}\left(\Delta_{c 1}\right) \\
\vdots \\
K_{c} C_{c d}\left(\Delta_{c 1}^{\prime}\right) \nu_{r_{1}}\left(\Delta_{c 1}\right)=\omega_{r_{1}}\left(\Delta_{c 1}\right)
\end{gathered}
$$

Step 2: Analysis performing a worst-case analysis (e.g. pole map or $\mu$-synthesis) for a finite number of closed loop LTI models in the parametric space (65 LTI closed loop models). If the synthesis meets control specifications for all the selected LTI models, then stop. Otherwise identify a worst-case model $G_{c d}\left(\Delta_{c(1+i)}\right)$ and continue to Step 3 .

Step 3: Multimodel synthesis improving the behavior of the LTI model identified in Step 2 respecting the specifications while preserving the closed loop properties of all the models treated before. The gain $K_{c}$ is designed considering the multimodel constraints of the previous step and those corresponding to $G_{c d}\left(\Delta_{c(1+i)}\right)$ :

$$
\begin{gathered}
K_{c} C_{c d}\left(\Delta_{c(1+i)}^{\prime}\right) \nu_{1}\left(\Delta_{c(1+i)}\right)=\omega_{1}\left(\Delta_{c(1+i)}\right) \\
\vdots \\
K_{c} C_{c d}\left(\Delta_{c(1+i)}^{\prime}\right) \nu_{r_{(1+i)}}\left(\Delta_{c(1+i)}\right)=\omega_{r_{(1+i)}}\left(\Delta_{c(1+i)}\right)
\end{gathered}
$$

This iterative procedure allows the calculation of the matrices $K_{y}, K_{z}, K_{i}$ required to satisfy robust performance and a stability when $\Delta_{c}$ deviates from the nominal model.

\section{RESULTS AND ANALYSIS}

The LPV model (4) is used to define a set of 65 LTI models corresponding to the number of characterized microspheres. The pole map of the open loop LPV system is shown in Fig.8. From the set of the 65 LTI models, the nominal model is selected such that its dominant pole has the highest real value (i.e. LTI model with the highest response time). This model is called $G_{c d}\left(\Delta_{c 1}\right)$. It corresponds to the $8^{\text {th }}$ microsphere of the reference 461 DET 40 d25.

\subsection{Simulation}

As defined in eq. (9), the triplet $\left\{U_{o}, T_{o}, \Pi_{o}\right\}$ of the observer is related to the matrices $A_{c d}\left(\Delta_{c 1}\right)$ and $C_{c d}\left(\Delta_{c 1}^{\prime}\right)$ of a nominal model $G_{c d}\left(\Delta_{c 1}\right)$. Therefore, when the system deviates from the nominal conditions $\left(\Delta_{c}\right.$ is different from $\Delta_{c 1}$ ) the relation (9) is no longer valid. As a 


$$
\begin{aligned}
\gamma_{j}\left(\Delta_{c i}\right) & =\left(\lambda_{j}\left(\Delta_{c i}\right)-\Pi_{o}\right)^{-1}\left(U_{o} B_{c d} \omega_{j}\left(\Delta_{c i}\right)-T_{o} C_{c d}\left(\Delta_{c i}^{\prime}\right) \nu_{j}\left(\Delta_{c i}\right)\right) \\
{\left[\begin{array}{lll}
K z & K y & K i
\end{array}\right] } & =\left[\begin{array}{lll}
\omega_{1}\left(\Delta_{c 1}\right) & \cdots & \omega_{r_{q}}\left(\Delta_{c q}\right)
\end{array}\right]\left[\begin{array}{ccc}
\gamma_{1}\left(\Delta_{c 1}\right) & \cdots & \gamma_{r_{q}}\left(\Delta_{c q}\right) \\
C_{c d}\left(\Delta_{c 1}^{\prime}\right) \nu_{1}\left(\Delta_{c 1}\right) & \cdots & C_{c d}\left(\Delta_{c q}^{\prime}\right) \nu_{r_{q}}\left(\Delta_{c q}\right)
\end{array}\right]^{-1}
\end{aligned}
$$

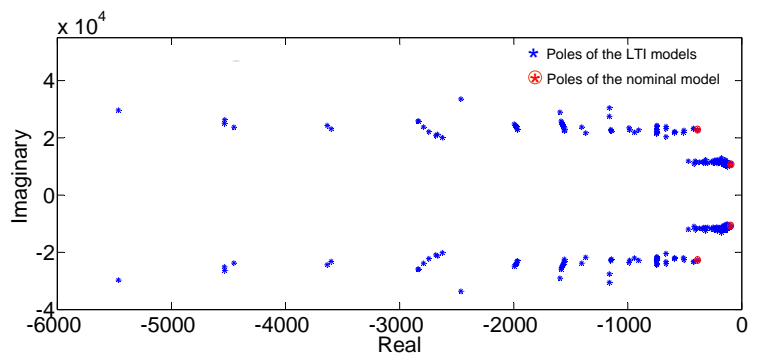

Fig. 8. Pole map of the open loop LPV system $G_{c d}\left(\Delta_{c}\right)$.

consequence, during the Step 3 (Multimodel synthesis), the eigenstructures of the worst case model $G_{c d}\left(\Delta_{c(1+i)}\right)$ can not be assigned exactly to the desired positions. In order assign precisely the eigenstructures of the worst case model $G_{c d}\left(\Delta_{c(1+i)}\right)$ while preserving the closed loop eigenstructures of the models treated before, we propose here to compute a self-scheduled form of the observer.

The order of the controller is equal to the number of observers. In order to obtain a low order controller, only dominant poles of the treated LTI models $G_{c d}\left(\Delta_{c i}\right)$ will be assigned at each step.

Initialization: The $E S A$ with the observer is applied on the nominal model $G_{c d}\left(\Delta_{c 1}\right)$. The number of assigned poles is $r_{1}=4$ (Table 2). Therefore, two observers have been designed considering lemma 2 . The observer is defined as:

$$
U_{o}=T_{o} C_{c d}\left(\Delta_{c 1}^{\prime}\right)\left(\Pi_{o} I-A_{c d}\left(\Delta_{c 1}\right)\right)^{-1}
$$

where $T_{o}=\left[\begin{array}{ll}1 & 1\end{array}\right]^{T}$ and $\Pi_{o}=10^{4} \times \operatorname{diag}(-2.75,-1.75)$.

The parameter $\Pi_{o}$ is related to the response time of the observer. It is defined such that it's smaller than the eigenvalue assigned by the output $Z$.

The gain $K_{c}=\left[\begin{array}{lll}K_{y} & K_{z} & K_{i}\end{array}\right]$ is computed considering eq. (18). The desired closed loop eigenvalues are: $-300,-500$, $-2 \times 10^{3}$ and $-3 \times 10^{3}$.

Analysis: The worst case analysis (see the pole map in Fig.9.a) shows that stability is not satisfied in the entire set. The pole map shows that a single step is not sufficient to satisfy robust performance and stability and validates the need of a multimodel assignment (Step 3). The worst case model $G_{c d}\left(\Delta_{c 2}\right)$ is defined such that its closed loop poles have the highest real value. It corresponds to the $11^{\text {th }}$ ball of the reference $461 \mathrm{WE} 20 \mathrm{~d} 36$.

Multimodel synthesis: the ESA with the observer is applied to the LTI models $G_{c d}\left(\Delta_{c 1}\right)$ and $G_{c d}\left(\Delta_{c 2}\right)$. The number of assigned poles is $r_{1}=4$ and $r_{2}=2$ (Table 2). Therefore, four observers have been designed, as assigning $r_{1}+r_{2}=6$ eigenstructures requires the use of (6-2) observers. The observer is defined such as:

$$
U_{o}=\left[\begin{array}{c}
U_{o}\left(\Delta_{c 1}\right) \\
U_{o}\left(\Delta_{c 2}\right)
\end{array}\right], T_{o}=\left[\begin{array}{c}
T_{o 1} \\
T_{o 2}
\end{array}\right], \Pi_{o}=\left[\begin{array}{cc}
\Pi_{o 1} & 0_{2 \times 2} \\
0_{2 \times 2} & \Pi_{o 2}
\end{array}\right]
$$

with:

$$
U_{o}\left(\Delta_{c 1}\right)=T_{o 1} C_{c d}\left(\Delta_{c 1}^{\prime}\right)\left(\Pi_{o 1} I-A_{c d}\left(\Delta_{c 1}\right)\right)^{-1}
$$
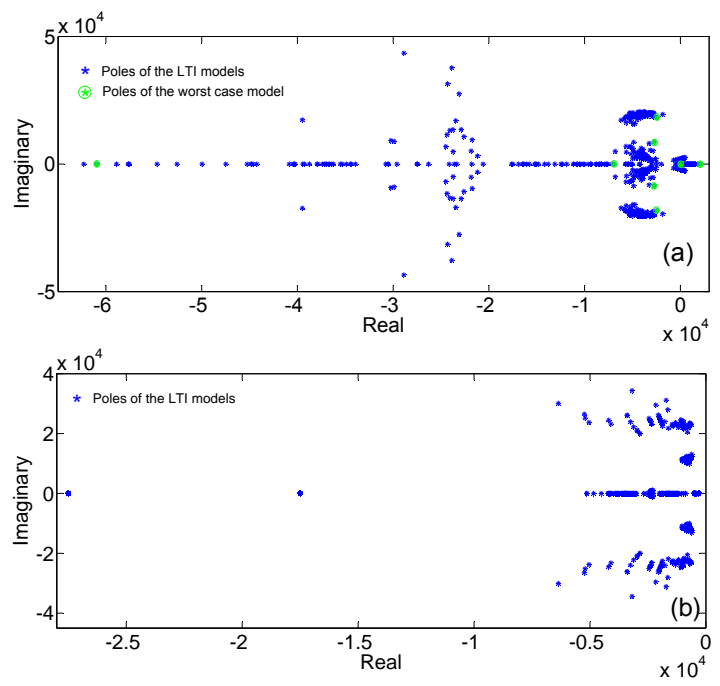

Fig. 9. Pole map obtained during the first step (a) and the third step (b).

$$
U_{o}\left(\Delta_{c 2}\right)=T_{o 2} C_{c d}\left(\Delta_{c 2}^{\prime}\right)\left(\Pi_{o 2} I-A_{c d}\left(\Delta_{c 2}\right)\right)^{-1}
$$

where: $T_{o 1}=T_{o 2}=\left[\begin{array}{ll}1 & 1\end{array}\right]^{T}$ and $\Pi_{o 1}=\Pi_{o 2}=10^{4} \times$ $\operatorname{diag}(-2.75,-1.75)$.

The gain $K_{c}=\left[\begin{array}{lll}K_{y} & K_{z} & K_{i}\end{array}\right]$ is computed considering eq. (18) and (19). The desired closed loop eigenvalues are: $-300,-500,-2 \times 10^{3}$ and $-3 \times 10^{3}$ for $G_{c d}\left(\Delta_{c 1}\right)$ and $-300,-400$ for $G_{c d}\left(\Delta_{c 2}\right)$.

Table 2 shows closed loop assigned eigenvalues of the models $G_{c d}\left(\Delta_{c 1}\right)$ and $G_{c d}\left(\Delta_{c 2}\right)$ during step 1 (analysis) and step 3 (multimodel synthesis). In step 3 , the eigenvalues of the model $G_{c d}\left(\Delta_{c 2}\right)$ are assigned precisely and the eigenvalues of the model $G_{c d}\left(\Delta_{c 1}\right)$ are the same as the ones assigned in step 1, preserving the performance of the nominal model.

The worst case analysis (Fig.9.b) shows that control specifications are satisfied for all the LTI models. The dynamic output feedback controller is of order 4 .

The nonlinear coupled model (1) is simulated with this self-scheduled controller. The force output $F_{c d}$ is used as feedback signal. The reference gripping force $F_{c r}$ is $20 \mu \mathrm{N}$. Fig.10.a and .b show the controlled gripping force $F_{c d}$ obtained by the self-scheduled controller designed in step 1 and step 3 respectively. A voltage saturation $V_{i n}=140 \mathrm{~V}$ is applied at the input of the open loop model. Instabilities and large errors are clearly visible in Fig.10.a. These results confirm the worst case analysis and the paramount need of a robust controller. Thanks to final controller, among the 65 closed loop models, the worst response time is $7.9 \mathrm{~ms}$, there is no overshoot and no static error.

One of the main advantages of the control strategy is that it allows obtaining a low order controller. With traditional $\mathrm{LPV} / \mathrm{H} \infty$ designs, the order of the controller is equal to the order of the open loop system + the order of weighting 
Table 2. Eigenvalues of $G_{c d}\left(\Delta_{c 1}\right)$ and $G_{c d}\left(\Delta_{c 2}\right)$ controlled by the controllers derived from step 1 and step 3 . The sign ' $*$ ' defines the assigned eigenvalues.

\begin{tabular}{lll}
\hline \multirow{2}{*}{ Steps } & $\begin{array}{l}\text { Closed loop eigenvalues } \\
\text { of } G_{c d}\left(\Delta_{c 1}\right)\end{array}$ & $\begin{array}{l}\text { Closed loop eigenvalues } \\
\text { of } G_{c d}\left(\Delta_{c 2}\right)\end{array}$ \\
\hline \hline Step 1 & $-300^{*}$ & $2.085 \times 10^{3}$ \\
& $-500^{*}$ & 4.43 \\
& $-2 \times 10^{3 *}$ & $-2.43 \times 10^{3}+1.82 \times 10^{4} i$ \\
& $-3 \times 10^{3 *}$ & $-2.43 \times 10^{3}-1.82 \times 10^{4} i$ \\
& $-3.75 \times 10^{3}+2.05 \times 10^{4} i$ & $-2.73 \times 10^{3}+8.57 \times 10^{3} i$ \\
& $-3.75 \times 10^{3}-2.05 \times 10^{4} i$ & $-2.73 \times 10^{3}-8.57 \times 10^{3} i$ \\
& $-1.75 \times 10^{4}$ & $-6.96 \times 10^{3}$ \\
& $-2.75 \times 10^{4}$ & $-6.09 \times 10^{4}$ \\
\hline Step 2 & $-300^{*}$ & $-300^{*}$ \\
& $-500^{*}$ & $-400^{*}$ \\
& $-2 \times 10^{3 *}$ & -876.5 \\
& $-3 \times 10^{3 *}$ & $-915.7+1.18 \times 10^{4} i$ \\
& $-628+2.29 \times 10^{4} i$ & $-915.7-1.18 \times 10^{4} i$ \\
& $-628-2.29 \times 10^{4} i$ & $-2.84 \times 10^{3}+2 \times 10^{4} i$ \\
& $-760.6+1.06 \times 10^{4} i$ & $-2.84 \times 10^{3}-2 \times 10^{4} i$ \\
& $-760.6-1.06 \times 10^{4} i$ & $-5.13 \times 10^{3}$ \\
& $-1.75 \times 10^{4}$ & $-1.75 \times 10^{4}$ \\
$-2.75 \times 10^{4}$ & $-2.75 \times 10^{4}$ \\
\hline
\end{tabular}
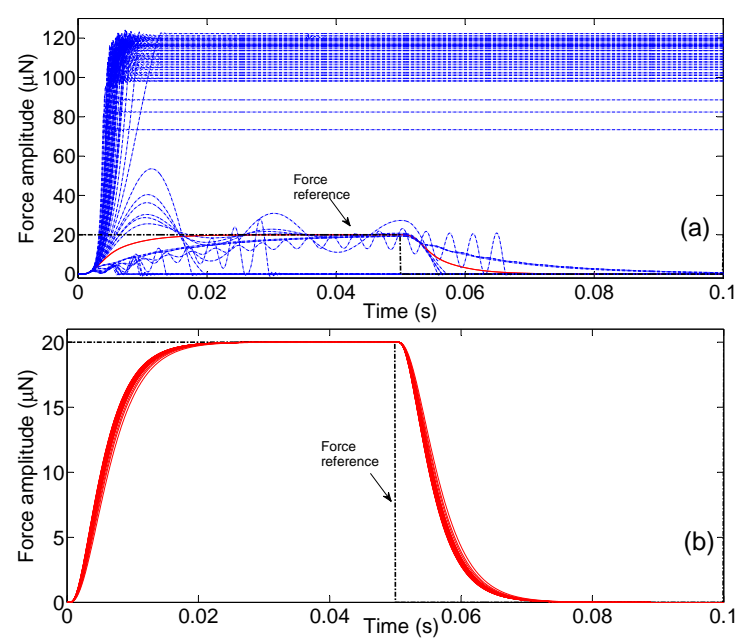

Fig. 10. Step responses $(20 \mu \mathrm{N}$ gripping force reference) of the controlled microgripper when gripping 65 different expancel microspheres: results from step 1 (a) and step 3 (b). The step responses satisfying control specifications are in solid line.

functions. With the coupled microgripper, the order of the controller would be at least equal to 6 (in the case of the use of a single first order weighting function).

\section{CONCLUSION}

This paper has dealt with stability and robustness issues of gripping force control at the microscale, using a nonlinear electrostatic microgripper with an integrated force sensor and a set of soft microspheres. The diameter and both the stiffness and the damping of the microspheres have been identified experimentally and have been considered uncertain parameters for the controller synthesis. The gripping force control has been achieved through the design of a robust self-scheduled controller based on an eigenstructure assignment methodology and a worst case analysis. The control methodology allows designing a low dimensional dynamic control scheme suitable for a real time implementation. Results emphasize the importance of the controller for safety manipulation tasks at the microscale which is of primary importance for a range of biological application.

\section{ACKNOWLEDGMENTS}

This work has been supported by the French national project NanoRobust- -(ANR 2011 NANO 006).

\section{REFERENCES}

F. Beyeler, A. Neild, S. Oberti, D. Bell, S. Yu, J. Dual, and B. J. Nelson. Monolithically fabricated microgripper with integrated force sensor for manipulating microobjects and biological cells aligned in an ultrasonic field. Journal of MEMS, 16:7-15, 2007.

A. Bolopion, H. Xie, S. Haliyo, and S. Régnier. Haptic teleoperation for $3 \mathrm{~d}$ microassembly of spherical objects. IEEE/ASME T- Mech, 17(1):116-127, 2012.

M. Boudaoud, Y. Le Gorrec, Y. Haddab, and P. Lutz. Gain scheduled control strategies for a nonlinear electrostatic microgripper: Design and real time implementation. In IEEE CDC, Maui, Hawaii, USA, 2012.

M. Boudaoud, Y. Haddab, and Y. Le Gorrec. Modeling and optimal force control of a nonlinear electrostatic microgripper. IEEE/ASME T- Mech, 18:1130-1139, 2013.

M. Boukallel, M. Girot, and S. Régnier. Characterization of cellular mechanical behavior at the microscale level by a hybrid force sensing device. Journal of the mechanical behavior of biomedical materials, 2(3):297-304, 2009.

M. C. Carrozza, A. Eisinberg, A. Menciassi, D. Campolo, S. Micera, and P. Dario. Towards a force-controlled microgripper for assembling biomedical microdevices. $J$. Micromech Microeng, 10:271-276, 2000.

D. Desmaele, M. Boukallel, and S. Régnier. A resonant structure designed for probing the elastic properties of suspension and adherent cells in liquid environments. $J$. Micromech Microeng, 22:115033 (9pp), 2012.

X. Liu, K. Kim, Y. Zhang, and Y. Sun. Nanonewton force sensing and control in microrobotic cell manipulation. Int J of Robotics Research, 28:1065-1076, 2009.

J. F. Magni, Y. Le Gorrec, and C. Chiappa. A multimodel based approach to robust and self-scheduled control design. In IEEE CDC, Tampa, FL, 1998.

B. C Moore and G Klein. Eigenvalue selection in the linear regulator combining modal and optimal control. In IEEE CDC, Florida, USA, 1976.

D. J. Muller, J. Helenius, D. Alsteens, and Y. F. Dufrene. Force probing surfaces of living cells to molecular resolution. Nat Chem Biol, 5, june 2009.

J. Park, S. Kim, D. H. Kim, B. Kim, S. J. Kwon, J. O Park, and K. I Lee. Identification and control of a sensorized microgripper for micromanipulation. IEEE/ASME TMech, 10:601-606, 2005.

C. Poussot-Vassal, O. Sename, L. Dugard, P. Gaspar, Z. Szabo, and J. Bokor. A new semi-active suspension control strategy through lpv technique. Control Engineering Practice, 16:1519-1534, 2008.

M. Rakotondrabe and Y. Le Gorrec. Force control in piezoelectric microactuators using self scheduled h $\infty$ technique cambridge, usa. In Symp on Mech Sys, 2010.

M. Rakotondrabe, Y. Haddab, and P. Lutz. Modelling and h-inf force control of a nonlinear piezoelectric cantilever. IEEE Int Conf on Int Robots and Systems, 2007. 